\title{
Thermal Improvable Behavior of A Pressed Fitting Mixed Explosive
}

\author{
Jia Lin, Zhang Lin-jun, Zhang Dong-mei, Gu Yan, Xin Kai-di, Chen Zhi-qun, \\ Luan Jie-yu, Wang Ke-yong, Chen-zhu, Zhao Juan, Sun Xu-dong, Zhou \\ Wen-jing \\ Xi'an Modern Chemistry Research Inst., Xi'an, 710065, China \\ email: 1169855351@qq.com
}

\begin{abstract}
Keywords: physical chemistry; hexogen; paraffin; mixed explosive; thermal improvable behavior
\end{abstract}
\begin{abstract}
A mixed explosive contains $\mathrm{Al}$ powder, hexogen (RDX), paraffin, etc. It is pressed fitting into $\Phi 20 \times 20 \mathrm{~mm}$ pillars. The pillars are heated in 55C for 57days and are measured in volume, mass and density. They are experimented in mechanical properties, sensitivities, stability. The micro-structure of the pillar are tested, and the outer skin of pillar is observed. The results reveal that pillar remains unchanged in mass, becomes bigger in volume and smaller in average density; Pillars show no cracks, but they become bigger in density-difference; pillars become bigger in both compression strength and shear strength, their vacuum stability test gas-bleed volume and friction sensitivity all becomes smaller; the paraffin in heated pillar is more regular, and RDX is better in quality. The above findings confirm that after heated, the paraffin in the pillars becomes attached to each other, and fills up the crevices between solid particles, i.e. its cohesion and cladding improve. And RDX improves in quality, so the pillars show a tendency of improvement in terms of macro property. All this shows that the thermal stress exercises a improvable function on the pillars.
\end{abstract}

\section{Introduction}

Since the energetic materials are autocatalytically discomposable and the temperature increase promotes the speed of decomposition, the study of thermal behavior of explosives \& propellants is focused on chemical change such as thermal decomposition and relatively little attention is paid to their physical change[1-5]. The physical change caused by mild thermal stress may result in structural and property change. It helps promote the safety of charging, storing and using powder to study the physical structure and chemical composition of powder charge and the changing law and mechanism of its thermal behavior of macro property.

This paper studies the changes of macro property and micro structure of a pressed fitting explosive pillar after it is heated in 55C to find out its law of thermal behavior.

\section{Experiments}

\section{Samples}

The mixed explosive contains $\mathrm{Al}$ powder, hexogen (RDX) and paraffin. The crushed paraffin is mixed with $\mathrm{Al}$ powder and RDX and then they are pressed into $13 \Phi 20 \times 20 \mathrm{~mm}$ pillars marked with A-1\#, A-2\#, A-0 (5pillars), A-57 (5pillars) and A-T'. We take the outmost skin (the thickness approximately $0.5 \mathrm{~mm}$ ) from A-T' pillar and have it fastened between two glass slides and market A-T. All samples are wrapped up in Al plastic bags, sealed (See Fig.1 for samples of A-2\# and A-T; other samples are omitted) and heated in 55C oven for the stipulated days.

\section{Instruments}

Moscow BT-400 CT, FEI-Quanta-600FEG-field-emission SEM, OLYMPUS lext 3D OLS 4100 measuring laser microscope, RENISHAW inVia raman microscope, METTLER TOLEDO FP90 central processor Leica DM 2500M projectile microscope.

\section{Test Methods and Conditions}

The vernier caliper with $0.001 \mathrm{~mm}$ accuracy is used to measure the diameters (the average 
diameter is obtained from 3 measurements of the upper, middle and lower pillar) and the height (the average height is obtained from 3 measurements of each pillar's axis and sides). The balance with $0.0001 \mathrm{~g}$ accuracy is used to measure the mass. The average density is calculated according to the volume and mass.

The national military criteria GJB 772A-1997 are observed for all the methods of the compression strength-compression method, shear strength-double shear method, impact sensitivity-explosion probability method, friction sensitivity-explosion probability method and vacuum stability test (VST)-pressure sensor method.

\section{Test Results}

\section{Changes of Volume, Mass and Density}

Heat A-1\# and A-2\# for respectively 0, 14, 24 36, 43 and 57 days before taking them out and measuring the pillars' diameters, heights and masses, calculating their volumes and average densities. We see that the volumes of A-1\# and A-2\# slightly increase when they are heated for 13 days, but their volumes begin to become smaller and tend to become stable when the heating time prolongs (From Fig. 2). The masses of pillars remain basically unchanged. Because the density is obtained by mass being divided by volume, the change is opposite to volume.

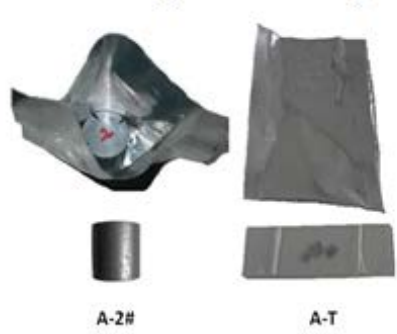

Fig.1 A-2\#, A-T and Their Al plastic bags

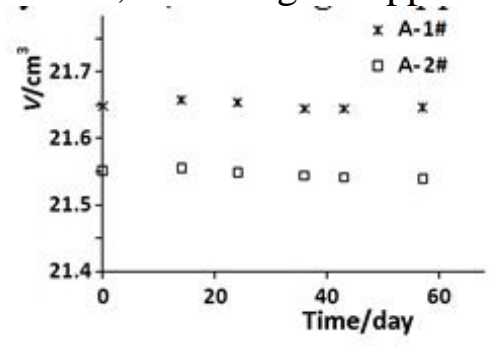

Fig.2 $V$ vs. time spectrum at $55 \mathrm{C}$

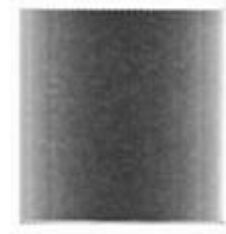

A-2\#(0day)

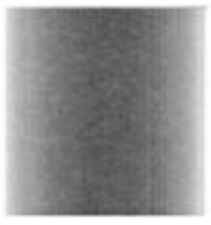

A-2\#(57day)

Test the density CT numbers of A-1\# and A-2\# after being heated for 0 and 57 days at the pillar axials $0^{\circ}$ and $90^{\circ}$. From Tab. 1 we see that, after being heated for 57 days, the CT numbers all become smaller, which means the pillars' average-density decreases. Their density-differences all increase, which means that the pillars' density differences increase on the same direction. This is due to the fact that the release of remaining stress at the pillar pressing results in increase of measurements, which leads to density decrease. The closer the place is to the outer edge of the pillar, the better the stress is released and the smaller the density is. Besides, we see from CT scanning pictures that no through flaws occur in the interior of A-1\# and A-2\# (Fig.3), which confirms that 57 days of 55C heating does not cause any structural damage to A-1\# and A-2\#.

Tab. 1 Test Results of Density CT Numbers

\begin{tabular}{lcccccccc}
\hline & \multicolumn{4}{c}{ Average-Density } & \multicolumn{3}{c}{ Density-Difference } \\
\cline { 2 - 8 } Time/day & \multicolumn{2}{c}{ Axial $0^{\circ}$} & \multicolumn{2}{c}{ Axial $90^{\circ}$} & \multicolumn{2}{c}{ Axial $0^{\circ}$} & \multicolumn{2}{c}{ Axial $90^{\circ}$} \\
\cline { 2 - 8 } & $1^{\#}$ & $2^{\#}$ & \multicolumn{2}{c}{$1^{\#} 2^{\#}$} & $1^{\#}$ & $2^{\#}$ & $1^{\#}$ & $2^{\#}$ \\
\hline 0 & 6809 & 6798 & 6845 & 6783 & $6.70 \%$ & $6.20 \%$ & $6.58 \%$ & $6.87 \%$ \\
\hline 57 & 6679 & 6606 & 6722 & 6643 & $6.71 \%$ & $6.98 \%$ & $6.94 \%$ & $6.94 \%$ \\
\hline
\end{tabular}

\section{Change of Mechanical Property, Sensitivity and Stability}

A- 0 is kept in room temperature and A-57 heated for 57 days. The test results are listed in Tab.2. From Tab. 2 we find that the pillars' compression strength and shear strength increase after heating, the impact sensitivity remains zero, the friction sensitivity becomes smaller and the gas release of VST decreases. All this shows that the pillars' mechanical properties, sensitivity and stability all improve after 57 days of heating.

Tab. 2 Test Results of Mechanical Properties, Sensitivity and Stability

\begin{tabular}{cccccc}
\hline Sample & $\begin{array}{c}\text { Compression Strength } \\
\text { /MPa }\end{array}$ & $\begin{array}{c}\text { Shear Strength } \\
\text { /MPa }\end{array}$ & $\begin{array}{c}\text { Impact Sensitivity } \\
/ \%\end{array}$ & $\begin{array}{c}\text { Friction Sensitivity } \\
/ \%\end{array}$ & $\begin{array}{c}\text { VST } \\
\mathrm{ml} / \mathrm{g}\end{array}$ \\
\hline A-0 & 4.91 & 1.39 & 0 & 8 & 0.06 \\
\hline A-57 & 6.36 & 2.55 & 0 & 0 & 0.05 \\
\hline
\end{tabular}




\section{Change of Micro Structure}

The sections of A-0 and A-57 which have been tested in shear strength are detected with SEM, laser microscope and raman microscope (Fig.4 6).

Fig.4 show that the paraffin in A-57 is more regular. We use projectile microscope to observe the change of heated paraffin after A-T is heated for 7 days in the $55 \mathrm{C}$ oven (Fig.7). We see that the light-transmission area increases (since the two glass slides cannot well fasten the A-T fragments, the A-T undergoes some shift in position before it is put into the oven after 0day of observation). We use projectile microscope to observe the change of pure paraffin heated from $40 \mathrm{C}$ to $67 \mathrm{C}$. We find that it begins to soften at 55C, to melt at $60 \mathrm{C}$ and becomes liquid at $66.6 \mathrm{C}$ ( Fig.8). The paraffin undergoes similar change in pillars. These pictures show that after heating the paraffin of the pillars softens and coheres, and then fills up the crevices between the solid granules. It is in the form of regular fragments.

From Fig.5 we see that the $\mathrm{Al}$ powder on $\mathrm{A}-57$ is more than that on $\mathrm{A}-0$. This confirms above words, that it is just because the paraffin on A-0 is scattered, crushed and is not mutually cohesive, that even the section on which there is little $\mathrm{Al}$ powder easily breaks. But the paraffin of A-57 is mutually cohesive, so only the section with more powdered Al breaks up.

To test the paraffin content on the outer layer of A-0 and A-57 pillars, two parallel samples are tested for each, $6.6 \%$ and $6.2 \%$ for $\mathrm{A}-0$ and $6.0 \%$ and $5.7 \%$ for $\mathrm{A}-57$. It shows that the outer layer paraffin is reduced after 57 days of heating, but we know the masses of pillars remain basically unchanged, so the analysis indicates that solids like RDX need to release the remaining stress and may "extend" outward, while the paraffin on the outer layer cohere with the paraffin on the inner layer due to softening.

Fig.6 shows that half-peak breadth values of RDX raman characteristic peaks (345、605、885、 $1312 \mathrm{~cm}^{-1}$ ) in A-57 are both smaller than RDX in A-0. This means that RDX crystals improve in quality after 57 days of heating.

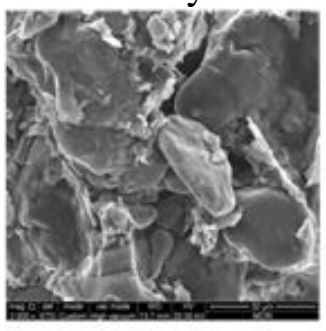

A-0

Fig.4 Scanning Electronic Microscope Pictures

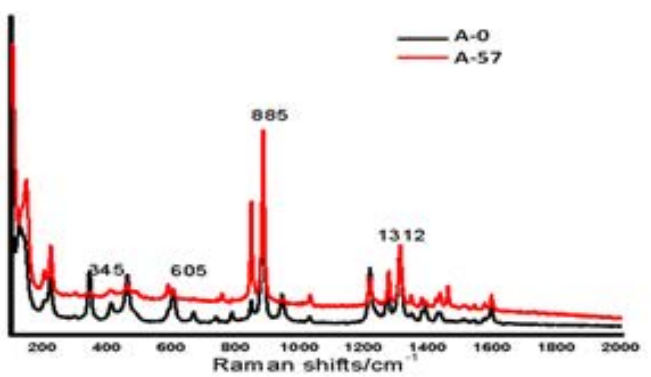

Fig.6 Raman Spectrums of RDX in A-0 and A-57

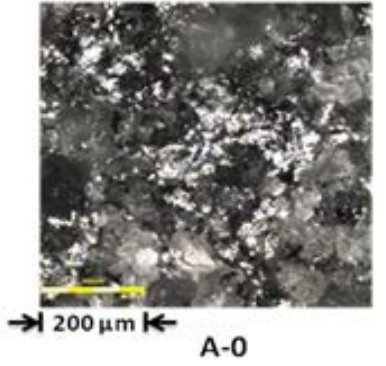

Fig.5 Laser Microscope Spectrums

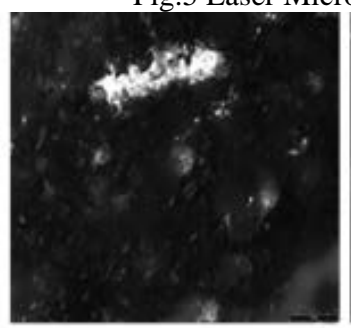

A-T(Oday)

Fig.7 Projectile Microscope Pictures of A-T

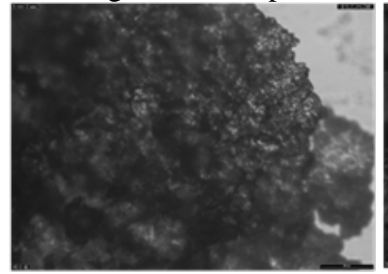

$40.0^{\circ} \mathrm{C}$

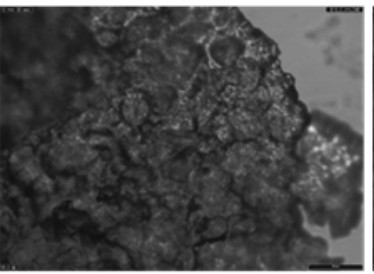

$55.0^{\circ} \mathrm{C}$

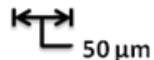

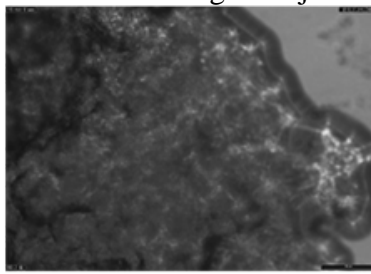

$60.0^{\circ} \mathrm{C}$

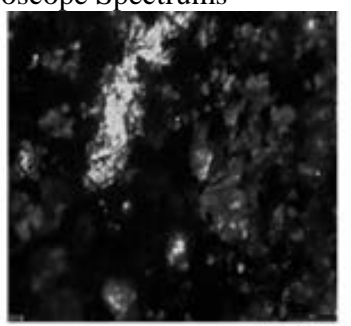

A-T(7day)

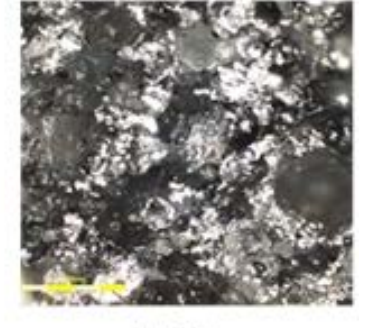

A-57

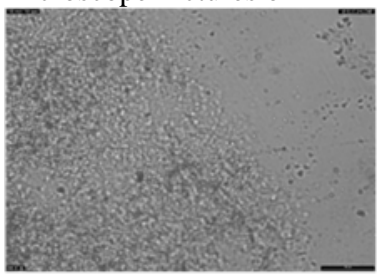

$66.6^{\circ} \mathrm{C}$

Fig.8. Pictures Showing the Change of Heated Paraffin 


\section{Conclusion}

From the analysis of the above we come to the following conclusion. After 57 days of 55C heating, fragmented paraffin begins to soften, gradually coheres and fills up the crevices between solids, so that its cladding and cohesive function improves. The RDX quality is enhanced also. These can explain why the pillars, after being heated, suffer no structural damage, but improve in their mechanical properties, sensitivity and stability. Therefore, we argue that 57 days of 55C heating is more of a "thermal improvable" role for the $\Phi 20 \times 20 \mathrm{~mm}$ pressed fitting explosive pillars.

\section{References}

[1] Weitao Yang, Yuxiang Li, and Sanjiu Ying. Fabrication, thermoanalysis, and performance evaluaton studies on RDX-based microcellular combustible objects [J]. Propellants, Explosives, Pyrotechnics, 2014, 39(4): 568-573.

[2] Xiaogan Dai, Yushi Wen, Fenglei Huang, and Yimin Huang. Effect of temperature, density and confinement on deflagration to detonation transition of an HMX-based explosive [J]. Propellants, Explosives, Pyrotechnics, 2014, 39(4): 563-567.

[3] Jing Mei, Shu Yuanjie, Wang Jun, Ma Qing, Zhang Xiaoyu, Huang Yigang. Synthesis, crystal structure and thermal property of 1-amino-2,4-dinitroimidazole [J]. Chinese Journal of Energetic Materials, 2014, 22(4): 454-457.

[4] Lu Honglin, Ju Ronghui, Fan Xuezhong, Zhao Fengqi, Wang Bozhou. Influences of lead/copper on the thermal decomposition characteristics of DHT [J]. Chinese Journal of Explosives \& Propellants, 2014, 37(4): 60-63.

[5] Gao Dayuan, Tan Kaiyuan, Li Jingming, Wen Shanggang, He Songwei, Zhou Jianhua. Thermal decomposition characteristics of RG-1 explosive [J]. Chinese Journal of Explosives \& Propellants, 2013, 36(5): 35-40.

[6] Lang Chen, Xin Ma, Feng Lu, Junying Wu. Investigation of the cook-off processes of HMX-based mixed explosives[J]. Central European Journal of Energetic Materials, 2014, 11(2): 199-218. 